\title{
Assessment of Periodontal Status in Association with Gestational Diabetes Mellitus: A Case-Control Study
}

Priyam Mishra*, Bagavad Gita and Chandrasekaran SC

Sree Balaji Dental College, Chennai, Tamil Nadu, India

\begin{abstract}
Background: Few studies have specifically examined the relationship between periodontal disease and gestational diabetes mellitus (GDM). Type 1 or 2 diabetes increases the risk of developing periodontal disease, and periodontal disease may also increase the risk for type 2 diabetes. However, there is no study done so far in a Tamil population where the prevalence of Type II diabetes mellitus is $>17 \%$.
\end{abstract}

The objective of this study was to examine whether maternal periodontal disease is associated with GDM in a Tamil urban population.

Aim: The aims of the study were-

To assess the periodontal status in patients with and without GDM

To examine any association between maternal periodontal disease and GDM

Materials and methods: The study population comprised of 90 patients, out of which 30 were cases \& 60 were controls. All cases underwent a laboratory screening test for GDM between 24 to 30 weeks of gestation based on the recommendation of the Obstetricians and Gynaecologists. Women were screened for carbohydrate intolerance by performing a standard 1-hour, 50-g oral Glucose Challenge Test (GCT). If the glucose level was $>135 \mathrm{mg} / \mathrm{dl}$ (GCT positive), then women were diagnosed with GDM and were considered as cases. Controls were defined as women who passed the GCT i.e glucose levels less than $135 \mathrm{mg} / \mathrm{dl}$ at 24 to 30 weeks and did not have any other types of diabetes. To assess the periodontal status, a full-mouth periodontal examination, assessing the probing depth, clinical attachment level, bleeding on probing and gingival recession was performed on all study participants, using the WHO periodontal probe by a single trained examiner. Tests for proportions and associations were performed by using chi-square statistics. To compare the means, student's t-test was performed. Multiple logistic regression analysis was performed by incorporating other predictors like maternal age, BMI, hygiene score, family history of DM and gestational age apart from periodontitis.

Results: The results showed that only age of the mother OR $1.387(95 \% \mathrm{C} \mid 1.129,1.704)$, body mass index OR $1.190(95 \%$ C I 1.029, 1.377), and family history of DM OR3.98 (95\% C I 1.09, 14.49) were significant predictors of GDM. None of the periodontitis condition was found to be significant predictor of GDM.

Conclusion: The study did not show any positive correlation between GDM \& periodontal disease.

Keywords: Gestational diabetes; Oral health; Oral hygiene indexsimplified; Periodontal disease; Pregnancy

\section{Introduction}

Periodontitis refers to an inflammatory condition of the soft tissues surrounding the teeth (i.e., gingivitis) and the destruction of the supporting structures of the teeth, including the periodontal ligament, bone, cementum, and soft tissues (i.e., periodontitis) $[1,2]$. Though primarily a polymicrobial infection, the development periodontitis is now also associated with a number of risk factors like genetic and environmental. Periodontal disease is now associated with a bidirectional risk of systemic diseases such as cardiovascular diseases, diabetes mellitus, and adverse pregnancy outcomes [3].

Few studies have already suggested that pregnancy is a modifying factor of periodontal disease [4]. Increased vascularisation and gingival inflammation have been reported as a result of an increase in estrogen and progesterone levels during pregnancy, which also leads to changes in the oral microflora [5]. Host response to this infection mediates a complex cascade of tissue-destructive pathways which diabetes amplifies resulting in periodontal destruction. Periodontal disease also acts like a reservoir for gram negative anaerobic flora, lipopolysaccharides and inflammatory mediators $\left(\mathrm{PGE}_{2}\right.$ and $\mathrm{TNF}$ ) which can trigger or increase insulin resistance [6].
GDM is defined as any degree of glucose intolerance with onset or first recognition during pregnancy [7]. True GDM resolves during the postpartum period. However, as many as 50 percent of women who had GDM remain at risk of developing type $2 \mathrm{DM}$, so GDM is thought to be a harbinger of DM in later life [8]. The glycemic status between the $24^{\text {th }}$ and $30^{\text {th }}$ weeks of pregnancy should be checked because the placenta grows larger in second \& third trimester so it secretes more hormones (estrogen, cortisol \& human placental lactogen) thereby making cells more resistant to insulin.

Few studies have specifically examined the relationship between periodontal disease and gestational diabetes mellitus. Studies have

*Corresponding author: Priyam Mishra, Sree Balaji Dental College, Chennai, Tamil Nadu, India, Tel: 8688380323; E-mail: priyam2805@gmail.com

Received September 22, 2014; Accepted November 18, 2014; Published November 21, 2014

Citation: Mishra P, Gita B, Chandrasekaran SC (2014) Assessment of Periodontal Status in Association with Gestational Diabetes Mellitus: A Case-Control Study. J Diabetes Metab 5: 467 doi:10.4172/2155-6156.1000467

Copyright: $\odot 2014$ Mishra $P$, et al. This is an open-access article distributed under the terms of the Creative Commons Attribution License, which permits unrestricted use, distribution, and reproduction in any medium, provided the original author and source are credited. 
found that type 1 or 2 diabetes increases the risk of developing periodontal disease, and periodontal disease may also increase the risk for type 2 diabetes $[9,10]$

As of now, we have no studies assessing the relationship between GDM and periodontitis in Tamil Nadu, although the prevalence of GDM is increasing here. The incidence of GDM was found to be $16.55 \%$ in 2004. In the recent field study performed under the Diabetes in Pregnancy-Awareness and Prevention project, the prevalence of GDM was $17.8 \%$ in the urban, $13.8 \%$ in the semi urban and $9.9 \%$ in the rural areas [11].

Thus, the purpose of this study was to evaluate the periodontal status in subjects with GDM and in subjects without GDM.

The aims of the study were-

1. To assess the periodontal status in patients with and without GDM between $24^{\text {th }}$ and $30^{\text {th }}$ weeks of pregnancy.

2. To examine any association between maternal periodontal disease and GDM.

\section{Materials and Methods}

\section{Study population}

The study population comprised of 90 patients, out of which 30 were cases and 60 were controls. It was estimated that, at a ratio of one case to two controls, a total of 30 cases as well as 60 controls would be sufficient to detect an Odds Ratio (OR) of 3.00. Therefore, a total of 30 cases of GDM and 60 controls were recruited from Sree Balaji Dental College \& Hospital \& Primary Health Centre, Adyar, Chennai between October 2009 and August 2011. This multicentric study was approved by the ethical committee board of Sree Balaji dental college $\&$ hospital.

\section{Definition of cases and controls}

At the hospital, all pregnant women underwent a laboratory screening test for GDM between 24 to 30 weeks of gestation based on the recommendation of the Obstetricians and Gynaecologists. Women were screened for carbohydrate intolerance by performing a standard 1-hour, 50-g oral glucose challenge test (GCT). If the glucose level was $>135 \mathrm{mg} / \mathrm{dl}$ (GCT positive), then women were diagnosed with GDM and were considered as cases. Controls were defined as women who passed the GCT i.e glucose levels less than $135 \mathrm{mg} / \mathrm{dl}$ at 24 to 30 weeks and did not have any other types of diabetes.

\section{Inclusion criteria}

Pregnant women were asked to participate if they met all of the following criteria:

1) Complete GDM screening at gestational age between $24^{\text {th }}$ to $30^{\text {th }}$ weeks.

2) Greater than or equal to 18 years of age.

3) Agreed to sign the consent form.

\section{Exclusion criteria}

1) Pre-gestational diabetes

2) Any heart disorders

3) HIV patients

4) Patients who have undergone any periodontal treatment within the past 3 months

\section{5) Having $<20$ teeth.}

All eligible pregnant women who were diagnosed with GDM (cases) and without GDM (controls) were invited to take part in the study. All participants gave written informed consent. After consenting, the research staff conducted an in-person, structured questionnaire interview.

\section{Periodontal disease measurements and definitions}

A full-mouth periodontal examination was performed on all study participants, using a manual probe: the WHO periodontal probe \& included:

1. 6 point probing depth (PD)

2. Gingival recession

3. Clinical attachment loss (CAL)

4. Bleeding on probing (BOP)

$\mathrm{PD}$ was defined as the distance in millimeters from the gingival margin to the apical extent of the pocket. Gingival recession was determined by measuring the distance from the cemento-enamel junction to the gingival margin in millimeters. CAL was calculated from recession and PD measurements and represented the distance from the cemento-enamel junction to the most apical portion of the sulcus/pocket in millimeters. BOP was assessed when probing to the base of the sulcus, six surfaces per tooth, and was expressed as the percentage of bleeding sites over the total tooth surfaces. OHI-S index given by Greene and Vermillion 1964, was taken for all participants as itsbeen widely used in epidemiological studies for periodontal studies. Third molars were not included in the analysis. To eliminate interexaminer variability, all periodontal measurements were performed by a single, trained examiner. For the present study, the case definition for periodontitis was defined as the presence of any site exhibiting PD $\geq$ $4 \mathrm{~mm}$ or CAL $\geq 4 \mathrm{~mm}$. We also used the criteria from the study by Offenbecher et al. on periodontal disease and adverse pregnancy outcomes, which defined periodontal disease as any site with $\mathrm{PD} \geq 4$ $\mathrm{mm}$ and clinical $\mathrm{AL} \geq 3 \mathrm{~mm}$ and severe periodontal disease as at least four sites with $\mathrm{PD} \geq 5 \mathrm{~mm}$ and four sites with $\mathrm{CAL} \geq 3 \mathrm{~mm}$.

We also collected other data like: sociodemographic data (e.g., age, pre-pregnancy weight/height, and body mass index [BMI]); socioeconomic status (e.g., education and family income); family history (e.g., mother or sisters with diabetes); oral hygiene and habits (frequency of dental care visit, dental cleaning, brushing teeth, and flossing teeth).

\section{Statistical analysis}

Frequency and cross classifications were done. Tests for proportions and associations were performed by using chi-square statistics. To compare the means, t-test was performed. Diagrams were generated where ever needed. To identify the factors associated with GDM, we used multiple logistic regression. The dependent variable was GDM (yes $=1 / \mathrm{No}=0$ ). All the statistical significances were by $5 \%$ level of significance. All the analysis was performed using SPSS16.

\section{Results}

There were 30 study subjects with GDM and 60 were from NonGDM group. Table 1 shows the characteristics of the study subjects. More than $85 \%$ of the study subjects were from lower socio economic background. Thirty three percent in GDM group and 37\% in NonGDM group were with more than three bleeding teeth. No bleeding was 


\begin{tabular}{|c|c|c|c|}
\hline Characteristic & $\operatorname{GDM}(n=30)$ & Non GDM $(n=60)$ & $P$ value \\
\hline Mean Age of mother & 28 & 24 & $0.00^{*}$ \\
\hline Occupation ( house wife \%) & 87 & 97 & 0.09 \\
\hline Socio economic ( poor \%) & 83 & 88 & 0.52 \\
\hline Bleeding on probing (\%) & $\begin{array}{l}40 \\
27 \\
33\end{array}$ & $\begin{array}{l}50 \\
13 \\
37\end{array}$ & 0.29 \\
\hline Family history of DM (\%) & 43 & 18 & $0.01^{*}$ \\
\hline $\begin{array}{r}\text { Diagnosis (\%) } \\
\text { Gen.chronic gingivitis } \\
\text { Gen. chronic periodontiti } \\
\text { Localised chronic periodontitis }\end{array}$ & $\begin{array}{l}13 \\
50 \\
37\end{array}$ & $\begin{array}{l}13 \\
65 \\
22\end{array}$ & 0.30 \\
\hline Mean body mass index & 29.2 & 25.2 & $0.00^{*}$ \\
\hline Mean oral hygiene score & 1.75 & 1.88 & 0.57 \\
\hline Mean Blood glucose & 162 & 105 & $0.00^{*}$ \\
\hline Mean gestational age (weeks) & 27.6 & 26.6 & 0.63 \\
\hline
\end{tabular}

* Significant at $5 \%$ level

Table 1: Characteristics of study subjects.

\begin{tabular}{|c|c|c|c|}
\hline Characteristics & Odd's ratio & $95 \% \mathrm{Cl}$ & P-value \\
\hline Age of mother & 1.387 & $1.129,1.704$ & $0.002^{*}$ \\
\hline BMI & 1.190 & $1.029, \quad 1.377$ & $0.019^{*}$ \\
\hline OHI-S score & 0.601 & $0.295, \quad 1.227$ & 0.162 \\
\hline Family history of DM (yes) & 0.251 & $0.069, \quad 0.917$ & $0.037^{*}$ \\
\hline $\begin{array}{l}\text { Diagnosis } \\
\text { 1. } \begin{array}{l}\text { Generalized chronic } \\
\text { gingivitis }\end{array} \\
\text { 2. } \begin{array}{l}\text { Generalized chronic } \\
\text { periodontitis }\end{array} \\
\text { 3. } \begin{array}{l}\text { Localized chronic } \\
\text { periodontitis }\end{array} \\
\end{array}$ & $\begin{array}{l}1.000 \\
0.486 \\
0.543\end{array}$ & $\begin{array}{ll}0.067, & 3.518 \\
0.078, & 3.789\end{array}$ & $\begin{array}{l}0.475 \\
0.538\end{array}$ \\
\hline Gestational age (weeks) & 1.227 & $0.949, \quad 1.60$ & 0.128 \\
\hline
\end{tabular}

* Significant at $5 \%$ level

Table 2: Factors associated with gestational diabetes.

observed on $40 \%$ of subjects in GDM and 50\% in Non-GDM group. However there was no association between bleeding on probing and GDM status. Significantly higher proportion (43\%) of GDM group had history of DM compared to Non-GDM group (18\%). Univariate analysis indicated that there was no association between the type of periodontitis and GDM status. The mean body mass index was 29.2 in GDM group; significantly higher level compared to Non-GDM. Ten out of 30 in GDM group were obese (BMI >=30) whereas it was 4 out of 60 subjects in Non-GDM group. Oral hygiene scores were obtained by using the OHI-S index. The mean oral hygiene scores were similar in both groups. In GDM group it was 1.78 and that in Non-GDM group it was 1.88 . As expected the mean blood glucose levels were significantly higher in GDM group. As far as the gestational age was considered, it was around 27 weeks in both groups.

\section{Multiple logistic regression}

The results of logistic regression analysis are given in Table 2. As our aim was to see whether periodontitis was a causative factor for GDM, the GDM status was the binary outcome variable viz. GDM (yes $=1 / \mathrm{No}=0$ ). Multiple logistic regression analysis was performed by incorporating other predictors like age of mother, BMI, hygiene score, family history of DM, gestational age apart from periodontitis. The results showed that only age of the mother OR 1.387 (95\% C I 1.129, 1.704), body mass index OR 1.190 (95\% C I 1.029, 1.377), and family history of DM OR3.98 (95\% C I 1.09, 14.49) were significant predictors of GDM. The odds of becoming GDM in mothers with family history of DM is about four times higher compared to the odds of becoming GDM in mothers with no family history of DM. This odds ratio can be as high as 14 times and as low as 1.09 times after controlling the influences of other factors considered here. The higher the age of the mother, the higher the odds compared to the odds of mothers getting GDM in the lower age group. None of the periodontitis condition was found to be significant predictor of GDM.

\section{Discussion}

The aim of the study was to assess the periodontal status in association with GDM. This multicenter study had 30 cases (pregnant women with GDM) and 60 controls (pregnant women without GDM) with gestation age between 24 to 30 weeks, as the study population. The variables considered were: Family history of diabetes mellitus, BMI and Periodontal variables: Gingival recession, OHI-S, BOP, PPD \& CAL

The results of this study showed that periodontal disease is not significantly associated with GDM with P-value of 0.475 for generalized chronic periodontitis \& P-value of 0.538 for localized chronic periodontitis. A study done in 2008, found an increase of clinical periodontal disease in women with GDM compared to women without GDM, but this increase did not reach statistical significance [12]. However, a case-control study done in 2009 reported a positive correlation between periodontal disease \& GDM with statistical significance [13].

A statistically significant positive correlation was seen for GDM with variables like BMI, OR 1.190 (95\% C I 1.029, 1.377) P-value of 0.019 , age of the mother, OR 1.387 (95\% C I 1.129, 1.704) P-value of 0.002 and family history of diabetes mellitus, OR 3.98 (95\% C I 1.09 , 14.49) P-value of 0.037. Risk factors for gestational diabetes mellitus include older maternal age, a higher body mass index (ADA, Xiong et al., Keshavarz et al., Dudhbhai et al.), prior gestational diabetes mellitus (ADA, Dudhbhai et al.), family history of diabetes among first-degree relatives (ADA, Keshavarz et al., Dudhbhai et al.), and being a member of a racial/ethnic group with a high prevalence of diabetes/GDM (ADA, 2004) [12]. Another study stated that pre pregnancy body mass index \& prior GDM are known risk factors for gestational diabetes mellitus [12]. Similarly Islam stated that obesity, measured by BMI predisposes women to complicated pregnancies \& increased obstetric interventions. Its a risk factor for developing GDM \& type II diabetes [14].

Several recent studies have suggested a relationship between periodontal disease and obesity. In Saito's study of Japanese adults, increasing body mass index and waist:hip ratio was associated with increasing risk of periodontitis. Data analyzed from the Third National Health and Nutrition Examination Survey reported a significant association between measures of body fat and periodontal disease among younger adults, but not among middle-aged or older adults [15]. A study reported correlations between body mass index, waist: hip ratio, and various periodontal measures, including mean periodontal attachment loss, mean pocket depth, mean gingival bleeding index, and mean calculus index [16]. Given recent evidence regarding adipose tissue serving as a reservoir for inflammatory cytokines, it is possible that increasing body fat increases the likelihood of an active host inflammatory response in periodontal disease [17].

Though our study did not show any positive correlation between GDM and periodontal disease, it did show an increased prevalence of periodontitis in controls. Gingivitis and poor oral hygiene is related to lower socio-economic status. This is most likely attributed to decrease in dental awareness and decreased frequency of dental visits compared 
with more educated individuals of higher socio-economic status [1]. The most likely explanation for the different results obtained in our study might be the smaller sample size which is not representative of a general population. Secondly, few other confounding variables like C-reactive proteins which have a role of inflammation in the etiology of gestational diabetes mellitus, presence of periodontal pathogens which may induce an inflammatory response and bacterial vaginitis have not been ruled out. In another study, the presence of periodontal pathogen, T.forsythia in vaginal flora as a potential risk factor for GDM was established [12]. An increased incidence of chorioamnionitis and premature rupture of membranes, which are clinical conditions associated with increased inflammatory cytokines and oxidative stress that can occur with gestational diabetes mellitus and increased oral T.forsythia colonization have also been reported [12].

Cohort studies may be able to throw more light on the elimination of bias of not knowing the previous periodontal status of the subjects. Whether periodontal disease is a causal risk factor for GDM or a result of GDM remains to be determined. It is well established that periodontal disease is more prevalent in individuals with type 1 or 2 diabetes compared to healthy controls $[10,18]$. Periodontitis has been referred to as the sixth complication of diabetes [9] and this has been attributed to consequences of a long duration of elevated blood glucose levels (hyperglycemia). Impaired insulin resistance, vascular changes, altered oral microflora, abnormal collagen metabolism, and the consequent hyperglycemia and hyperlipidemia of diabetes result in metabolic alterations that exacerbate bacteria-induced inflammatory periodontitis.

GDM only represents an early stage of glucose dysregulation and a temporary impaired glucose tolerance that occur in later pregnancy. The elevated glucose levels in the majority of women diagnosed with GDM usually return to normal after birth [19]. Therefore, the hyperglycemia of GDM may be too mild and of too short a duration to have a significant effect on gingival tissues and to cause a destruction of the supporting structures (i.e., tissue and bone) of the teeth, which are manifested as periodontitis. An alternative explanation is that periodontal disease may be a cause, instead of the result, of GDM. Periodontal infection, a local and chronic subclinical inflammation, triggers a maternal systemic inflammatory response [20,21]. Because pregnancy itself is a stressful state with increased inflammatory activity [22] and marked insulin resistance [23], such an infection-induced insulin resistance in response to maternal periodontal infection may worsen the pre-existing pregnancy-induced insulin resistance that may cause impaired glucose tolerance and the manifestation of GDM [21].

Our study was powered with sufficient several important confounding variables (e.g., socioeconomic status, BMI, family history of diabetes, systemic antibiotics during Pregnancy \& oral hygiene and behaviours). GDM diagnosis was made by following the stricter guidelines of Carpenter and Coustan [24]. Because women with GDM are at higher risk of developing type 2 diabetes [25] and periodontal disease has been implicated as a risk factor for type 2 diabetes [10,18], periodontal disease may contribute to a the development of diabetes in women with a previous history of GDM [21].

\section{Conclusion}

Periodontal disease is preventable and curable. So, if periodontal disease is confirmed as risk factor for GDM in future studies, this might lead to intervention studies. Improving oral health and treating periodontal disease before or during pregnancy might reduce maternal and infant morbidity associated with GDM during pregnancy and prevent type 2 diabetes later in life. The physician and periodontist should make a combined effort to educate the patients regarding the implications of poor oral hygiene on preterm low birth weight deliveries even before pregnancy and encourage periodic assessment of the oral hygiene status.

\section{References}

1. [No authors listed] (1996) Position paper: epidemiology of periodontal diseases American Academy of Periodontology. J Periodontol 67: 935-945.

2. Kinane DF (2001) Causation and pathogenesis of periodontal disease Periodontol 2000 25: 8-20.

3. Xiang AH, Peters RK, Trigo E, Kjos SL, Lee WP, et al. (1999) Multiple metabolic defects during late pregnancy in women at high risk for type 2 diabetes. Diabetes 48: 848-854.

4. Laine MA (2002) Effect of pregnancy on periodontal and dental health. Acta Odontol Scand 60: 257-264.

5. Ruiz DR, Romito GA, Dib SA (2011) Periodontal disease in gestational and type 1 diabetes mellitus pregnant women. Oral Dis 17: 515-521.

6. Nishimura F, Soga Y, Iwamoto Y, Kudo C, Murayama Y (2005) Periodonta disease as part of the insulin resistance syndrome in diabetic patients. J Int Acad Periodontol 7: 16-20.

7. Zargar AH, Wani AA, Laway BA, Masoodi SR, Wani Al, et al. (2008) Prevalence of diabetes mellitus and other abnormalities of glucose tolerance in young adults aged 20-40 years in North India (Kashmir Valley). Diabetes Res Clin Pract 82: 276-281.

8. Kidambi S, Patel SB (2008) Diabetes mellitus: considerations for dentistry. $J$ Am Dent Assoc 139 Suppl: 8S-18S

9. Löe H (1993) Periodontal disease. The sixth complication of diabetes mellitus Diabetes Care 16: 329-334

10. Soskolne WA, Klinger A (2001) The relationship between periodontal diseases and diabetes: an overview. Ann Periodontol 6: 91-98.

11. Seshiah V, Balaji V, Balaji MS, Paneerselvam A, Arthi T, et al. (2008) Prevalence of gestational diabetes mellitus in South India (Tamil Nadu)--a community based study. J Assoc Physicians India 56: 329-333.

12. Dasanayake AP, Chhun N, Tanner ACR, Craig RG, Lee MJ, et al. (2008) Periodontal Pathogens and Gestational Diabetes Mellitus. J dent res 87: 328.

13. Islam A, Khan NA, Ehsan A. Complications of raised BMI in pregnancy Professor Med J Sep 17: 498-504.

14. Al-Zahrani MS, Bissada NF, Borawskit EA (2003) Obesity and periodontal disease in young, middle-aged, and older adults. J Periodontol 74: 610-615.

15. Wood N, Johnson RB, Streckfus CF (2003) Comparison of body composition and periodontal disease using nutritional assessment techniques: Third National Health and Nutrition Examination Survey (NHANES III). J ClinPeriodontol 30: 321-327.

16. Greenberg AS, Obin MS (2006) Obesity and the role of adipose tissue in inflammation and metabolism. Am J Clin Nutr 83: 461S-465S.

17. Lamster IB, Lalla E, Borgnakke WS, Taylor GW (2008) The relationship between oral health and diabetes mellitus. J Am Dent Assoc 139 Suppl: 19S-24S.

18. Buchanan TA, Xiang AH (2005) Gestational diabetes mellitus. J Clin Invest 115 485-491.

19. Garcia RI, Henshaw MM, Krall EA (2001) Relationship between periodontal disease and systemic health. Periodontol 2000 25: 21-36.

20. Xiong X, Buekens P, Fraser WD, Beck J, Offenbacher S (2006) Periodontal disease and adverse pregnancy outcomes: a systematic review. BJOG 113: 135-143.

21. Redman CW, Sacks GP, Sargent IL (1999) Preeclampsia: an excessive maternal inflammatory response to pregnancy. Am J Obstet Gynecol 180: 499506 .

22. Williams D (2003) Pregnancy: a stress test for life. Curr Opin Obstet Gynecol 15: $465-471$

23. Carpenter MW, Coustan DR (1982) Criteria for screening tests for gestational diabetes. Am J Obstet Gynecol 144: 768-773. 
Citation: Mishra P, Gita B, Chandrasekaran SC (2014) Assessment of Periodontal Status in Association with Gestational Diabetes Mellitus: A CaseControl Study. J Diabetes Metab 5: 467 doi:10.4172/2155-6156.1000467

Page 5 of 5

24. Kim C, Newton KM, Knopp RH (2002) Gestational diabetes and the incidence of type 2 diabetes: a systematic review. Diabetes Care 25: 1862-1868.
25. Matthews DC (2002) The relationship between diabetes and periodontal disease. J Can Dent Assoc 68: 161-164. 